Published in final edited form as:

Lancet Child Adolesc Health. 2019 January ; 3(1): 35-43. doi:10.1016/S2352-4642(18)30309-2.

\title{
Co-occurrence of early diabetes-related complications in adolescents and young adults with type 1 diabetes: the SEARCH for Diabetes in Youth study
}

Katherine A Sauder, $\mathrm{PhD}^{1}$, Jeanette M Stafford, $\mathbf{M S}^{2}$, Elizabeth J Mayer-Davis, $\mathrm{PhD}^{3}$, Elizabeth T Jensen, $\mathrm{PhD}^{4}$, Sharon Saydah, PhD $^{5}$, Amy Mottl, $\mathbf{M D}^{6}$, Lawrence M Dolan, MD ${ }^{7}$, Richard F Hamman, MD $^{8}$, Jean M Lawrence, ScD $^{9}$, Catherine Pihoker, MD $^{10}$, Santica Marcovina, PhD ${ }^{11}$, Ralph B. D'Agostino Jr, PhD ${ }^{2}$, Dana Dabelea, MD $^{1,8}$, and for the SEARCH for Diabetes in Youth Study Group

${ }^{1}$ Department of Pediatrics, University of Colorado School of Medicine, 13123 E. 16th Ave - B065, Aurora, CO 80045, USA

${ }^{2}$ Department of Biostatistical Sciences, Wake Forest School of Medicine, Medical Center Blvd, Winston-Salem, NC 27103, USA

${ }^{3}$ Departments of Nutrition and Medicine, University of North Carolina, 135 Dauer Drive, Chapel Hill, NC 27599, USA

${ }^{4}$ Department of Epidemiology and Prevention, Wake Forest School of Medicine, Winston-Salem, NC 27103, USA

${ }^{5}$ Division of Diabetes Translation, Centers for Disease Control and Prevention, 1600 Clifton Rd, Atlanta, GA 30329, USA

${ }^{6}$ Division of Nephrology and Hypertension, University of North Carolina School of Medicine, CB \#7155, Chapel Hill, NC 27599, USA

${ }^{7}$ Divison of Endocrinology, Cincinnati Children's Hospital Medical Center, 3333 Burnet Avenue, Cincinnati, OH 45229, USA

${ }^{8}$ Department of Epidemiology, Colorado School of Public Health, 13001 E 17th Place - B119, Aurora, CO 80045, USA

${ }^{9}$ Department of Research and Evaluation, Kaiser Permanente Southern California, 100 S. Los Robles Ave, Pasadena, CA 91101, USA

${ }^{10}$ Department of Pediatrics, University of Washington, 1900 Ninth Avenue, Seattle, WA 98101 , USA

Correspondence Katherine Sauder, University of Colorado School of Medicine, 12474 E 19th Avenue F426, Aurora CO, 80045, katherine.sauder@ucdenver.edu, Telephone: 303-724-8715.

Author Contributions. KAS, JMS, RBD, and DD designed the present research question, developed the analysis plan, and drafted the manuscript. EMD, ETJ, LD, JML, CP, SM, RBD, and DD supervised data collection. JMS and RD conducted the analyses. All authors interpreted the results, critically reviewed the manuscript for intellectual content, and approved the final manuscript. KAS takes responsibility for the contents of the manuscript.

Duality of Interest. Dr. D'Agostino Jr reports personal fees from Amgen and KCI outside the submitted work. Dr. Marcovina reports consulting for Denka Seiken and MedTest Dx outside the submitted work. The other authors declared no conflicts of interest. 
${ }^{11}$ Northwest Lipid Research Laboratory, University of Washington, 401 Queen Anne Ave N, Seattle, WA 98109, USA

\section{Abstract}

Background.-One in three adolescents and young adults with type 1 diabetes have at least one early diabetes-related complication/comorbidity. However, the prevalence, patterning, and risk factors for co-occurring complications in this population are not well understood.

Methods.-The SEARCH for Diabetes in Youth observational cohort study includes 1327 individuals diagnosed with type 1 diabetes before 20 years of age from 5 United States locations. Sociodemographic and metabolic risk factors were assessed at baseline (mean diabetes duration $=$ 0.8 years, mean age $=10.9$ years) and follow-up (mean diabetes duration $=7.8$ years, mean age $=$ 18.0 years). Early diabetes complications (diabetic kidney disease, diabetic retinopathy, peripheral neuropathy, cardiovascular autonomic neuropathy, and arterial stiffness) were assessed at followup. We aimed to describe co-occurrence of complications and examine differences in cooccurrence within demographic and metabolic risk factor clusters identified using cluster analysis.

Findings.-Overall, co-occurrence of any $\geq 2$ complications was observed in $5.9 \%$ of all participants, more frequently than expected by chance alone $(4.4 \%, \mathrm{p}=0 \cdot 015)$. Specifically, retinopathy and diabetic kidney disease, retinopathy and arterial stiffness, and arterial stiffness and cardiac autonomic neuropathy all co-occurred more frequently than expected (all $\mathrm{p}<0.05$ ). The cluster analysis produced four unique clusters characterized by progressively worsening metabolic risk factor profiles (longer duration; higher A1c, non-HDL cholesterol, and waist to height ratio) and differences in sociodemographic characteristics (race/ethnicity, household income, type of health insurance). Prevalence of $\geq 2$ complications progressively increased with worsening metabolic profiles (from $2.3 \%$ to $20.8 \%$, p $<0.001$ ).

Interpretation.-We report that early complications co-occur in adolescents and young adults with type 1 diabetes more frequently than expected after an average of less than eight years of diabetes duration. A cluster of high risk factors identifies groups that may benefit most from interventions to reduce complications.

\section{INTRODUCTION}

The burden of type 1 diabetes is continuing to increase worldwide. ${ }^{1}$ In the United States, the incidence of type 1 diabetes among youth <20 years of age increased by $1.4 \%$ per year from 2002 to $2012,{ }^{2}$ and the prevalence increased more than $20 \%$ from 2001 to $2009,{ }^{3}$ resulting in a growing number of individuals living with diabetes from youth onward. The presence of diabetes-related early complications and comorbidities in this population is substantial, with one in three individuals exhibiting early signs of any one complication/comorbidity after just eight years of average diabetes duration. ${ }^{4}$ As the duration of diabetes increases, the prevalence of each complication/comorbidity is expected to rise, particularly when accompanied by suboptimal glycemic control. ${ }^{5}$ Several studies have also reported that microvascular complications (retinopathy, neuropathy, nephropathy) tend to co-occur in individuals with type 1 diabetes, although this work has largely been conducted among adults aged 30 years or older with at least 15 years of diabetes duration. ${ }^{6-9}$ 
In younger populations, there is limited understanding of the co-occurrence of early complications and comorbidities within a relatively short duration of type 1 diabetes. In a Danish study of 339 participants aged 12-24 years, individuals with microalbuminuria at baseline were more likely to have both nephropathy and neuropathy, but not retinopathy, six years later. ${ }^{10}$ In contrast, a study of 80 Swedish participants aged 7-22 years reported that individuals with neuropathy were not more likely to simultaneously exhibit nephropathy or retinopathy compared to those without. ${ }^{11}$ Our group previously reported that type 1 diabetes participants who had cardiac autonomic neuropathy were significantly more likely to concurrently exhibit increased arterial stiffness at an average age of 18.6 years and $10 \cdot 1$ years of diabetes duration. ${ }^{12}$ While other studies have reported on prevalence of complications/comorbidities in younger populations with type 1 diabetes, ${ }^{13,14}$ these studies did not examine the prevalence of co-occurring complications, or the risk factors that are associated with co-occurring complications. Since co-occurring complications are associated with increased personal and societal burden, ${ }^{9,15}$ this knowledge can help identify youth at highest risk who may need early intensive intervention.

Thus, the purpose of this study was to examine the co-occurrence of diabetes-related early complications/comorbidities in a large, diverse sample of adolescents and young adults with type 1 diabetes. We examined co-occurrence by number and type, as well as by demographic characteristic and metabolic risk factor groups identified using cluster analysis. We hypothesized that there would be non-random co-occurrence of complications/comorbidities among participants that differed in their demographic characteristics and metabolic risk factors.

\section{METHODS}

\section{Participants.}

The present study includes data from a subset of individuals participating in the SEARCH for Diabetes in Youth Study. SEARCH identifies individuals diagnosed with any type of diabetes before 20 years of age through a population-based registry network at 5 sites in the United States (South Carolina; Cincinnati, Ohio and surrounding counties; Colorado with southwestern American Indian sites; Seattle, Washington, and surrounding counties; and Kaiser Permanente Southern California membership in 7 counties). Individuals who received a new diagnosis of type 1 or type 2 diabetes in 2002-2006 or 2008 were invited to complete a baseline SEARCH visit to measure risk factors for diabetes complications; by design, individuals diagnosed in 2007 were not invited to participate in baseline interviews. In 2011-2015, participants with $\geq 5$ years diabetes duration, who had previously completed a baseline visit, were invited to participate in a follow-up visit, at which diabetes risk factors and early diabetes-related complications and comorbidities were assessed. This follow-up visit was completed by 2777 participants at a mean age of 17.9 years (SD 4.8) and mean diabetes duration of $8 \cdot 0$ years (SD 2.0). The distribution of demographic, metabolic, and socioeconomic characteristics of participants who completed the follow-up visit were similar to that of the larger SEARCH registry population. ${ }^{4}$ The study was approved by the institutional review boards with jurisdiction in each study location. All participants provided 
consent or assent as age-appropriate, and parents also provided consent for those aged $<18$ years.

\section{Demographic, anthropometric, and metabolic assessments.}

Trained research staff conducted the in-person baseline and follow-up research visits. Participants (or parents, for younger participants) self-reported date of birth, sex, race, ethnicity, highest parental education, annual household income, and type of health insurance. Date of diagnosis had been obtained from medical records as part of the registry study, and was used to calculate age of diagnosis and diabetes duration at each visit. Height and weight were measured in light indoor clothing without shoes and used to calculate body mass index (BMI, $\mathrm{kg} / \mathrm{m}^{2}$ ) and age- and sex-specific BMI z-scores. ${ }^{16}$ Circumference at the natural waist was measured and used to calculate waist-to-height ratio. Blood pressure was measured after 5 minutes of seated rest, with the mean of three readings taken 1 minute apart used for analysis. Mean arterial pressure was calculated as $1 / 3$ systolic $+2 / 3$ diastolic. Participants were instructed to fast overnight for $\geq 8$ hours and abstain from medications (including shortacting insulin) on the morning of the visit. Blood samples were obtained and analyzed for hemoglobin A1c, glucose, lipids, creatinine, and cystatin $\mathrm{C}$ at the central laboratory (Northwest Lipid Metabolism and Diabetes Research, Seattle, WA). Participants were asked to bring a first morning urine void to the follow-up visit; if not done, a spot urine specimen was collected at the time of the visit ( 8\% of participants). Urine samples were analyzed for albumin and creatinine at the central laboratory.

\section{Diabetes classification.}

Baseline blood specimens were analyzed for glutamic acid decarboxylase- 65 antibodies and insulinoma-associated-2 antibodies at the central laboratory, ${ }^{17}$ and for zinc-T8 autoantibody at the Eisenbarth Laboratory (University of Colorado, Denver, CO). ${ }^{18}$ Insulin sensitivity at baseline was estimated using a validated equation that included waist circumference, hemoglobin A1c, and triglyceride levels. ${ }^{19}$ Etiologic type 1 diabetes was defined as $\geq 1$ positive antibody result (regardless of insulin sensitivity) or no positive antibody results and insulin sensitivity (score $\geq 8 \cdot 15$ ). ${ }^{20}$

\section{Diabetes-related complication/comorbidity assessments.}

Diabetic kidney disease was defined as the presence of albuminuria ( $230 \mu \mathrm{g} / \mathrm{mg}$ of creatinine) or low glomerular filtration rate ( $\ 60 \mathrm{~mL} / \mathrm{min} / 1 \cdot 73 \mathrm{~m}^{2}$ as estimated by the CKDEPI equations with serum creatinine and cystatin C). ${ }^{21}$ Diabetic retinopathy was assessed with $45^{\circ}$ color digital fundus images taken with a nonmydriatic camera (Visucam Pro N, Carl Zeiss Meditech) and centered on the disc and macula of both eyes. Photos masked to all clinical characteristics were graded by the Wisconsin Ocular Epidemiology Reading Center. Diabetic retinopathy was defined as mild, moderate, or proliferative retinopathy in at least one eye. ${ }^{22}$ Our threshold for diagnosing retinopathy was set higher than typically used clinically in order to distinguish diabetic retinopathy from milder forms that are possibly related to etiologies other than diabetes retinopathy. Peripheral neuropathy was defined as a score $>2$ on the Michigan Neuropathy Screening Instrument. ${ }^{23}$ Cardiovascular autonomic neuropathy was assessed by heart rate variability using the SphygmoCor-Vx device (AtCor Medical). Electrocardiographic R-R intervals measured in a supine position for 10 minutes 
were used to estimate five heart rate variability indices: the SD of the intervals, root mean square differences of successive intervals, normalized high-frequency power, normalized low-frequency power, and the low-to-high frequency ratio. Cardiovascular autonomic neuropathy was defined as abnormalities in three or more of the five indices, based on $2^{\text {th }}$ or $\geq 5^{\text {th }}$ percentile (as appropriate) observed in age- and sex-matched control participants of the SEARCH Cardiovascular Disease (CVD) ancillary study. ${ }^{12}$ Arterial stiffness was measured with the SphgymoCor-Vx device, and defined as a carotid-femoral pulse wave velocity $\geq 0^{\text {th }}$ percentile compared to control participants of the SEARCH CVD study. ${ }^{12}$

\section{Statistical analyses.}

Analyses were conducted in two parts. First, a frequency analysis was used to examine the difference in observed versus expected prevalence of co-occurring complications/ comorbidities. Second, a cluster analysis (Ward minimum variance method) was used to identify unique clusters of participants based on demographic characteristics and metabolic risk factors, between whom the prevalence of co-occurring complications/comorbidities was compared. A sensitivity analysis was performed for the cluster analysis by winsorizing the data by limiting the extreme values to 3 standard deviations within the mean value in order to reduce the potential impact of outliers on the Ward method for cluster analysis. Results were compared between these different approaches. All analyses were conducted in SAS v9.4 (Cary, NC) with statistical significance set at 0.05.

The frequency analysis included 1327 SEARCH participants who had etiologic type 1 diabetes, were age $\geq 10$ years, reported using insulin and had complete data on all five complications/comorbidities of interest at the follow-up visit (Figure 1). For these participants, we classified prevalence of co-occurring complications/comorbidities in multiple ways: a) discrete number of complications/comorbidities for each participant (0-5), b) mutually exclusive numerical category of co-occurring complications (none, only one, any 2 or more), c) type of complication/comorbidity occurring singly (e.g., retinopathy and no others), and d) all possible pairs of co-occurring complications/comorbidities (e.g., retinopathy + diabetic kidney disease, regardless of the presence or absence of other complications/comorbidities). For each classification, we used exact binomial tests to determine if the observed prevalence significantly differed from the prevalence expected by chance alone. ${ }^{24}$ Expected cell counts were calculated by examining the overall contingency table of all possible combinations of complications/comorbidities and using this table to calculate the individual expected cell counts (proportions) assuming independence across all cells. These expected cell counts (proportions) allowed us to construct specific exact binomial tests to determine whether the observed proportions were different from the expected proportions. The advantage of using exact binomial tests for comparing observed to expected proportions is that this approach is appropriate for each comparison regardless of the cell count observed within any particular cell of the table.

The cluster analysis was restricted to 1142 of the above participants with type 1 diabetes who had complete data for sex, race/ethnicity, age at the follow-up visit, and the following metabolic risk factor variables from both the baseline and follow-up visits: duration of diabetes, hemoglobin A1c, waist to height ratio, mean arterial pressure, HDL cholesterol, 
and non-HDL cholesterol (Figure 1). We used cluster analysis to identify unique clusters of participants based on these characteristics. We compared demographics, metabolic characteristics, and numerical category of co-occurring complications/comorbidities between clusters using the Chi-Square test for categorical variables or Kruskal-Wallis tests for continuous variables. Although ANOVA models would be valid to compare most continuous variables, the Kruskal-Wallis test was used to allow the same test to be used for all variables even if the assumption of normality were not met for all variables.

\section{RESULTS}

Participant characteristics are reported in Table 1. On average, participants were 10.1 years old at the time of type 1 diabetes diagnosis, completed their baseline visit at 10.9 years of age ( 0.8 years diabetes duration), and completed their follow-up visit at 18.0 years of age (7.8 years diabetes duration). The majority of participants (76.6\%) were non-Hispanic white. At baseline, the majority of participants had parents with a high school education or more (95.9\%), lived in households with annual incomes $\$ \$ 50,000$ (59.9\%), and had private health insurance $(80.3 \%)$. The average hemoglobin A1c was $7.6 \%(60.0 \mathrm{mmol} / \mathrm{mol})$ at baseline and $9.2 \%(76.8 \mathrm{mmol} / \mathrm{mol})$ at the follow-up visit. In general, blood pressure and lipid levels were higher at the follow-up visit compared to the baseline visit.

The prevalence and co-occurrence of early type 1 diabetes-related complications/ comorbidities is reported in Table 2. Overall, $70.9 \%$ of participants had no complications/ comorbidities, which was not significantly different than what was expected by chance alone $(68.4 \%, \mathrm{p}=0.053)$. Fewer participants than expected had one and only one complication/ comorbidity ( $23.2 \%$ versus $27.2 \%, \mathrm{p}=0.0011)$, while more participants than expected had any two or more $(5.9 \%$ versus $4.4 \%, \mathrm{p}=0 \cdot 015)$. Only two participants had four complications/comorbidities, and none of the participants had all five. The prevalence of each complication/comorbidity occurring in isolation was lower from that expected by chance, although this was statistically significant only for retinopathy (1.8\% versus $2.9 \%$, $\mathrm{p}=0.016$ ). Pair-wise comparisons showed that retinopathy + diabetic kidney disease, retinopathy + arterial stiffness, and arterial stiffness + cardiovascular autonomic neuropathy each co-occurred more frequently than expected (all $\mathrm{p}<0.05$ ). The remaining pair-wise comparisons also reflected cooccurrence more frequently than expected, although they did not reach statistical significance.

The cluster analysis identified four unique participant clusters of varying sizes, which were labeled according to the severity of metabolic risk factors (low risk $n=261$, mid risk $n=509$, high risk $n=348$, highest risk $n=24$; Table 3 ). The sensitivity analysis using the winsorized data (i.e., data limited to within 3 standard deviations of the mean) also generated four clusters with 1120 out of 1142 participants being classified into the same clusters using either method. The 22 participants who were re-classified were all in the third (high) cluster in the original analyses, with nine being considered in the mid-risk cluster and 13 being considered in the higher-risk cluster. Since the cluster analysis results are very similar using both methods, we present the main results below using the non-winsorized data since it represented the actual observed data for the participants; the results using the winsorized data are presented in the Appendix. 
As expected, the clusters significantly differed for nearly all the variables used to derive them (sex, race/ethnicity, age at follow-up visit, hemoglobin A1c at follow-up visit, baseline and follow-up values of duration of diabetes, waist to height ratio, mean arterial pressure, HDL cholesterol, and non-HDL cholesterol; all p<0.05). The comparison of baseline hemoglobin A1c did not reach statistical significance ( $\mathrm{p}=0.088$ ), although the point estimates suggest that the participants in the higher risk clusters had the highest A1c. They also differed significantly in the proportion of participants who had private health insurance and household incomes $\$ 50,000$ annually. Moving across clusters from lowest to highest risk, there were significant increases in diabetes duration (baseline $\mathrm{p}=0 \cdot 018$, follow-up $\mathrm{p}=0 \cdot 0028$ ), hemoglobin A1c (follow-up $\mathrm{p}<0 \cdot 0001$ ), waist to height ratio (baseline and follow-up p <0.0001), and non-HDL cholesterol (baseline and follow-up p $<0.0001$ ). For both mean arterial pressure and HDL cholesterol at baseline, values worsened from low to mid to high risk ( $\mathrm{p}=0.0051$ and $\mathrm{p}<0.0001$, respectively); however the highest risk cluster had the lowest mean arterial pressure and highest HDL cholesterol of all the clusters at baseline. At the follow-up visit, however, mean arterial pressure increased and HDL cholesterol decreased from the low to highest risk clusters (all $\mathrm{p}<0 \cdot 001$ ). Although these comparisons are not a priori, per se, since they are made conditional on the specific result of the cluster analysis, we note that of the 15 variables that we compared across clusters, twelve had pvalues that were less than 0.01 and eight of these were less than $0 \cdot 001$, indicating that the differences are quite strong, and the notable difference in means across clusters were substantial, indicating the effects are quite large (e.g., A1c at follow-up of 8.5 versus 11.8 in the low and highest risk clusters, respectively). The clusters notably differed in terms of the number of co-occurring complications/comorbidities ( $p<0.001$; Figure 2 ). There was a decrease across clusters in the proportion of participants with no complications/ comorbidities $((78.9 \%, 69.5 \%, 66.1 \%$, and $41.7 \%)$, and an increase in the proportion with any 2 or more $(2 \cdot 3 \%, 6 \cdot 3 \%, 8 \cdot 0 \%, 20 \cdot 8 \%)$.

\section{DISCUSSION}

In this cohort of adolescents and young adults with type 1 diabetes, we observed a higher than expected co-occurrence of any two or more early diabetes-related complications at a relatively young age and short duration of diabetes. Complications occurring in isolation were observed less frequently than expected, indicating that adolescents and young adults with type 1 diabetes who develop any complication are likely to have concurrent complications. Individuals with high-risk profiles at baseline and after eight years of followup were ten times more likely to develop multiple complications than individuals with less adverse profiles. These data suggest that there are subgroups of young people with type 1 diabetes, identifiable within the first few years of diabetes duration, for whom efforts to prevent diabetes-related complications beginning soon after diagnosis may be particularly needed.

Co-occurring complications are not uncommon among older adults with a longer duration of type 1 diabetes: there have been reports of an increased prevalence of retinopathy with neuropathy, ${ }^{6-8}$ retinopathy with nephropathy, ${ }^{6,23}$ and neuropathy with nephropathy, ${ }^{7}$ as well as the "triopathy". ${ }^{25}$ Increased arterial stiffness and/or cardiovascular autonomic neuropathy have also been observed at higher frequencies among adults also exhibiting retinopathy, ${ }^{26}$ 
neuropathy, ${ }^{8,12}$ or nephropathy. ${ }^{26}$ In adolescent and young adult populations with type 1 diabetes, a higher prevalence of retinopathy with nephropathy ${ }^{14}$ and cardiovascular autonomic neuropathy with arterial stiffness ${ }^{12}$ have been reported. Our study confirms these latter adolescent and young adult results, and provides new evidence that retinopathy is likely to co-occur with arterial stiffness in this population. We also showed that retinopathy was significantly less likely to occur individually, as just 24 of the 54 participants had retinopathy only. Our data suggest that adolescent and young adult patients who screen positive even for early stages of diabetic retinopathy are likely to have another complication, emphasizing the importance of regular screening for complications in line with current recommendations. ${ }^{27}$

Our study provides novel evidence of the higher than expected burden of any two or more co-occurring complications in adolescents and young adults with type 1 diabetes. This is likely due to common or overlapping etiologies for the complications. The clusters that we identified using demographic and metabolic characteristics at baseline and after eight years of follow-up demonstrated that there are early and persistent risk factors that can distinguish between individuals who are significantly more or less likely to develop multiple complications after a relatively short disease duration. Compared to participants with the least adverse metabolic risk factor profile, those with the most adverse metabolic risk profile were half as likely to be free of complications at follow-up and ten times as likely to develop any two or more complications. There was a consistent trend of worsening characteristics across clusters for most risk factors that was evident at both baseline and follow-up. The change in risk factors over time was also disparate between clusters: in the lowest risk cluster, metabolic risk factors changed little from baseline to follow-up, while the highest risk cluster exhibited substantial increases in hemoglobin A1c, mean arterial pressure, and non-HDL cholesterol, and decreases in HDL cholesterol. These data are consistent with prior studies that report metabolic risk factors for complications in type 1 diabetes, including suboptimal glycemic control, ${ }^{5,10,13}$ hypertension, ${ }^{6,8,21}$ and dyslipidemia. ${ }^{4}$ The relatively high A1c levels we observed at the follow-up visit (average of 9.2\%) are particularly worrisome and serve as a call to action to improve glycemic control of adolescents and young adults with type 1 type 1 diabetes in the United States. In line with current clinical recommendations, ${ }^{27}$ our data indicate that intensive management of cardiometabolic risk factors among individuals with type 1 diabetes, in addition to improving glycemic control, could be vital to preventing early onset of multiple complications. Moreover, our cluster analysis suggests heterogeneity in the population of individuals with type 1 diabetes with a substantial proportion (32.6\% of our sample) disproportionately at risk for co-occurring complications.

The clusters also differed according to sex, race/ethnicity, type of health insurance, and household income. The proportion of females increased from the lowest to highest risk cluster, suggesting that co-occurring complications are more likely to occur in girls and young women. Prior studies of co-occurring complications in type 1 diabetes have not reported on sex differences, ${ }^{6-8,10,11,14}$ although studies of individual complications have reported either a higher prevalence among males, ${ }^{6,10,28}$ or females, ${ }^{29}$ or no sex differences. 7,13,14 Despite lower incidence and prevalence of type 1 diabetes in Hispanic, non-Hispanic black, Asian/Pacific Islander, or American Indian youth compared to non-Hispanic white 
youth, 2,3 the clusters with the fewest non-Hispanic white youth had a higher prevalence of co-occurring complications. Prior studies have documented racial/ethnic disparities in microvascular complications of type 1 diabetes, which have been attributed to biological, behavioral, social, environmental, and healthcare factors. ${ }^{30}$ The clusters with a lower proportion of private health insurance and annual household income also exhibited a greater prevalence of co-occurring complications, even though these variables were not included in the analysis used to generate the clusters. These data collectively suggest that non-white youth from low-resource environments who have adverse metabolic profiles are more likely to develop co-occurring early complications. However, it is notable that some participants $(2.3 \%)$ in the low risk cluster did develop $\geq 2$ complications by follow-up, while over $40 \%$ in the high risk cluster did not develop any complications by follow-up. Further research is needed to understand the factors operating in the participants with high risk profiles that may protect from the development of co-occurring complications, as well as the factors that contribute to the manifestation of co-occurring complications among youth with apparently low risk profiles.

This study has strengths and limitations. We used a large, racially and ethnically diverse sample that is clinically and demographically representative of the overall population of adolescents and young adults with type 1 diabetes in the United States. ${ }^{4}$ Longitudinal measurements of sociodemographic and metabolic risk factors are a strength, while a single assessment of early diabetes complications without confirmatory testing is a limitation. The number of participants with co-occurring complications was small for some combinations, including those with three or more complications, which prevented a more detailed examination of co-occurring frequencies and patterns. Lastly, our results may have limited generalizability to populations with other degrees of glycemic control, with different sociodemographic characteristics, or differences in clinical management of type 1 diabetes.

In conclusion, we report that early diabetes complications/comorbidities co-occur more frequently than expected among adolescents and young adults with type 1 diabetes at a relatively short diabetes duration. A cluster of high-risk factors identifies groups that could potentially be targeted by behavioral or medical interventions to reduce the early development of life-long debilitating diabetes-related morbidity.

\section{Supplementary Material}

Refer to Web version on PubMed Central for supplementary material.

\section{Acknowledgements.}

The SEARCH for Diabetes in Youth Study is indebted to the many youth and their families, and their health care providers, whose participation made this study possible.

The authors wish to acknowledge the involvement of the South Carolina Clinical \& Translational Research Institute, at the Medical University of South Carolina, NIH/National Center for Advancing Translational Sciences (NCATS) grant number UL1 TR000062; Seattle Children's Hospital and the University of Washington, NIH/ NCATS grant number UL1 TR00423; University of Colorado Pediatric Clinical and Translational Research Center, NIH/NCATS grant Number UL1 TR000154; the Barbara Davis Center at the University of Colorado at Denver (DERC NIH grant number P30 DK57516); the University of Cincinnati, NIH/NCATS grant number UL1 TR000077; and the Children with Medical Handicaps program managed by the Ohio Department of Health. This 
study includes data provided by the Ohio Department of Health, which should not be considered an endorsement of this study or its conclusions.

The findings and conclusions in this report are those of the authors and do not necessarily represent the official position of the Centers for Disease Control and Prevention or the National Institute of Diabetes and Digestive and Kidney Diseases.

Funding. Grant Support: SEARCH for Diabetes in Youth is funded by the Centers for Disease Control and Prevention (PA numbers 00097, DP-05-069, and DP-10-001) and supported by the National Institute of Diabetes and Digestive and Kidney Diseases.

Site Contract Numbers: Kaiser Permanente Southern California (U48/CCU919219, U01 DP000246, and U18DP002714), University of Colorado Denver (U48/CCU819241-3, U01 DP000247, and U18DP000247-06A1), Children's Hospital Medical Center (Cincinnati) (U48/CCU519239, U01 DP000248, and 1U18DP002709), University of North Carolina at Chapel Hill (U48/CCU419249, U01 DP000254, and U18DP002708), University of Washington School of Medicine (U58/CCU019235-4, U01 DP000244, and U18DP002710-01), Wake Forest University School of Medicine (U48/CCU919219, U01 DP000250, and 200-2010-35171).

Role of the Sponsors. Dr. Saydah (CDC) is a participating member of the study Steering Committee and the Writing Group for this manuscript because of the cooperative funding agreement. She was involved in the design of the study but not the conduct of the study; she was not involved in the collection, management, and analysis of the data, but was involved in interpretation of the data; she was involved in the preparation, review, and approval of the manuscript and the decision to submit the manuscript for publication.

Funding. Centers for Disease Control and Prevention, National Institutes of Health

\section{References}

1. Diamond Project Group. Incidence and trends of childhood Type 1 diabetes worldwide 1990-1999. Diabet Med 2006; 23(8): 857-66. [PubMed: 16911623]

2. Mayer-Davis EJ, Lawrence JM, Dabelea D, et al. Incidence Trends of Type 1 and Type 2 Diabetes among Youths, 2002-2012. N Engl J Med 2017; 376(15): 1419-29. [PubMed: 28402773]

3. Dabelea D, Mayer-Davis EJ, Saydah S, et al. Prevalence of type 1 and type 2 diabetes among children and adolescents from 2001 to 2009. JAMA 2014; 311(17): 1778-86. [PubMed: 24794371]

4. Dabelea D, Stafford JM, Mayer-Davis EJ, et al. Association of Type 1 Diabetes vs Type 2 Diabetes Diagnosed During Childhood and Adolescence With Complications During Teenage Years and Young Adulthood. JAMA 2017; 317(8): 825-35. [PubMed: 28245334]

5. Microvascular and acute complications in IDDM patients: the EURODIAB IDDM Complications Study. Diabetologia 1994; 37(3): 278-85. [PubMed: 8174842]

6. De Block CE, De Leeuw IH, Van Gaal LF. Impact of overweight on chronic microvascular complications in type 1 diabetic patients. Diabetes Care 2005; 28(7): 1649-55. [PubMed: 15983315]

7. Dyck PJ, Kratz KM, Karnes JL, et al. The prevalence by staged severity of various types of diabetic neuropathy, retinopathy, and nephropathy in a population-based cohort: the Rochester Diabetic Neuropathy Study. Neurology 1993; 43(4): 817-24. [PubMed: 8469345]

8. Zander E, Seidlein I, Herfurth S, et al. Increased prevalence of proliferative retinopathy and cardiovascular autonomic dysfunction in IDDM patients with proteinuria. Exp Clin Endocrinol 1992; 99(2): 102-7. [PubMed: 1639116]

9. Morgan CL, Currie CJ, Stott NC, Smithers M, Butler CC, Peters JR. The prevalence of multiple diabetes-related complications. Diabet Med 2000; 17(2): 146-51. [PubMed: 10746486]

10. Olsen BS, Sjolie A, Hougaard P, et al. A 6-year nationwide cohort study of glycaemic control in young people with type 1 diabetes. Risk markers for the development of retinopathy, nephropathy and neuropathy. Danish Study Group of Diabetes in Childhood. J Diabetes Complications 2000; 14(6): 295-300. [PubMed: 11120452]

11. Nordwall M, Hyllienmark L, Ludvigsson J. Early diabetic complications in a population of young patients with type 1 diabetes mellitus despite intensive treatment. J Pediatr Endocrinol Metab 2006; 19(1): 45-54. [PubMed: 16509528] 
12. Jaiswal M, Urbina EM, Wadwa RP, et al. Reduced heart rate variability is associated with increased arterial stiffness in youth with type 1 diabetes: the SEARCH CVD study. Diabetes Care 2013; 36(8): 2351-8. [PubMed: 23435158]

13. Demirel F, Tepe D, Kara O, Esen I. Microvascular complications in adolescents with type 1 diabetes mellitus. J Clin Res Pediatr Endocrinol 2013; 5(3): 145-9. [PubMed: 24072081]

14. Pedro RA, Ramon SA, Marc BB, Juan FB, Isabel MM. Prevalence and relationship between diabetic retinopathy and nephropathy, and its risk factors in the North-East of Spain, a populationbased study. Ophthalmic Epidemiol 2010; 17(4): 251-65. [PubMed: 20642348]

15. Brownrigg JR, Hughes CO, Burleigh D, et al. Microvascular disease and risk of cardiovascular events among individuals with type 2 diabetes: a population-level cohort study. Lancet Diabetes Endocrinol 2016; 4(7): 588-97. [PubMed: 27216886]

16. Kuczmarski RJ, Ogden CL, Grummer-Strawn LM, et al. CDC growth charts: United States. Adv Data 2000; (314): 1-27.

17. Bonifacio E, Yu L, Williams AK, et al. Harmonization of glutamic acid decarboxylase and islet antigen-2 autoantibody assays for national institute of diabetes and digestive and kidney diseases consortia. J Clin Endocrinol Metab 2010; 95(7): 3360-7. [PubMed: 20444913]

18. Lampasona V, Schlosser M, Mueller PW, et al. Diabetes antibody standardization program: first proficiency evaluation of assays for autoantibodies to zinc transporter 8. Clin Chem 2011; 57(12): 1693-702. [PubMed: 21980171]

19. Dabelea D, D'Agostino RB, Jr., Mason CC, et al. Development, validation and use of an insulin sensitivity score in youths with diabetes: the SEARCH for Diabetes in Youth study. Diabetologia 2011; 54(1): 78-86. [PubMed: 20886205]

20. Dabelea D, Pihoker C, Talton JW, et al. Etiological approach to characterization of diabetes type: the SEARCH for Diabetes in Youth Study. Diabetes Care 2011; 34(7): 1628-33. [PubMed: 21636800]

21. Bjornstad P, Cherney DZ, Maahs DM. Update on Estimation of Kidney Function in Diabetic Kidney Disease. Curr Diab Rep 2015; 15(9): 57. [PubMed: 26188736]

22. Klein R, Klein BE, Magli YL, et al. An alternative method of grading diabetic retinopathy. Ophthalmology 1986; 93(9): 1183-7. [PubMed: 3101021]

23. Feldman EL, Stevens MJ, Thomas PK, Brown MB, Canal N, Greene DA. A practical two-step quantitative clinical and electrophysiological assessment for the diagnosis and staging of diabetic neuropathy. Diabetes Care 1994; 17(11): 1281-9. [PubMed: 7821168]

24. SAS Version 9.4. PROC FREQ procedure with the EXACT option Cary, NC, USA: SAS Institute Inc; 2011.

25. Root HF, Pote WH, Jr., Frehner H. Triopathy of diabetes; sequence of neuropathy, retinopathy, and nephropathy in one hundred fifty-five patients. AMA Arch Intern Med 1954; 94(6): 931-41. [PubMed: 13217491]

26. Gordin D, Waden J, Forsblom C, et al. Arterial stiffness and vascular complications in patients with type 1 diabetes: the Finnish Diabetic Nephropathy (FinnDiane) Study. Ann Med 2012; 44(2): 196204. [PubMed: 21047152]

27. American Diabetes A. 10. Microvascular Complications and Foot Care. Diabetes Care 2017; 40(Suppl 1): S88-S98. [PubMed: 27979897]

28. Raile K, Galler A, Hofer S, et al. Diabetic nephropathy in 27,805 children, adolescents, and adults with type 1 diabetes: effect of diabetes duration, A1C, hypertension, dyslipidemia, diabetes onset, and sex. Diabetes Care 2007; 30(10): 2523-8. [PubMed: 17630266]

29. Goni MJ, Forga L, Ibanez B, Cambra K, Mozas D, Anda E. Incidence and Risk Factors Involved in the Development of Nephropathy in Patients with Type 1 Diabetes Mellitus: Follow Up Since Onset. Can J Diabetes 2016; 40(3): 258-63. [PubMed: 26976719]

30. Spanakis EK, Golden SH. Race/ethnic difference in diabetes and diabetic complications. Curr Diab Rep 2013; 13(6): 814-23. [PubMed: 24037313] 


\section{Research in context}

\section{Evidence before this study}

In November 2017, we searched PubMed for English language publications with no date limitations using the following terms alone and in combination: type 1 diabetes, complications, co-occurrence, multiple, retinopathy, neuropathy, nephropathy, kidney disease, arterial stiffness, heart rate variability, pulse wave velocity, youth, children, and adolescents. We also reviewed reference lists of identified original and review articles. We found that studies examining diabetes-related complications/comorbidities among youth and young adults with type 1 diabetes, including our recent analysis (Dabelea et al, JAMA 2017), largely examined complications individually, and did not report whether complications/comorbidities were likely to co-occur, or the risk factors related to such co-occurrence.

\section{Added value of this study}

We evaluated the co-occurrence and related risk factors of multiple diabetes-related complications and comorbidities among youth and young adults with type 1 diabetes across five sites in the United States. We observed a higher than expected co-occurrence of any two or more early diabetes-related complications at a relatively young age and at only eight years of diabetes duration. Individuals with the highest risk profile at baseline and after eight years of follow-up were ten times more likely to develop multiple complications than individuals with less adverse profiles.

\section{Implications of all the available evidence}

One in three adolescents and young adults with type 1 diabetes have at least one early diabetes-related complication/comorbidity, and complications/comorbidities co-occur more frequently than expected by chance alone at a relatively short diabetes duration. A cluster of high risk factors identifies groups that may be targeted by interventions to reduce the early development of life-long debilitating diabetes-related morbidity. 


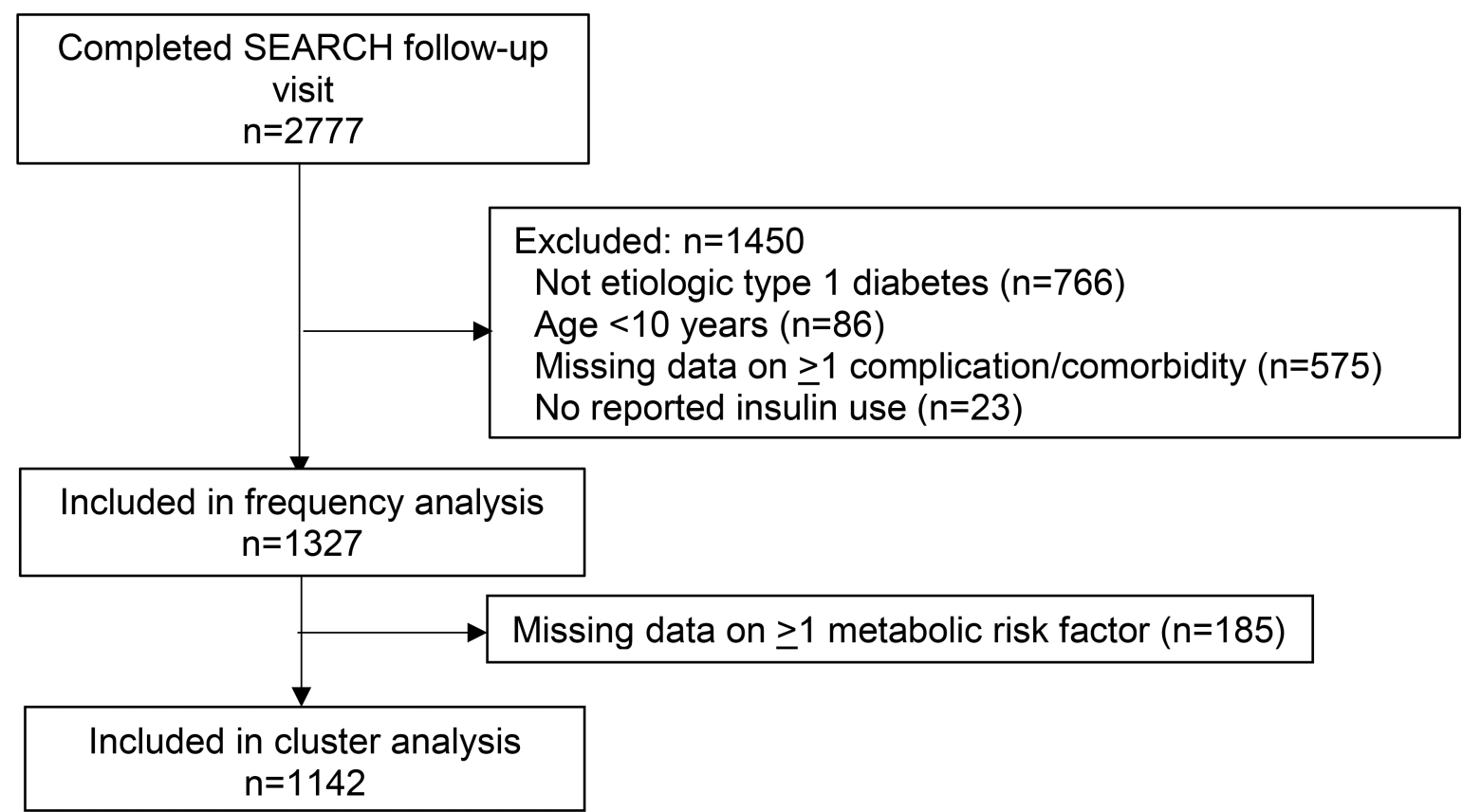

Figure 1.

Flow diagram of participants in the SEARCH for Diabetes in Youth Study at the time of the follow-up visit (2010-2015) 


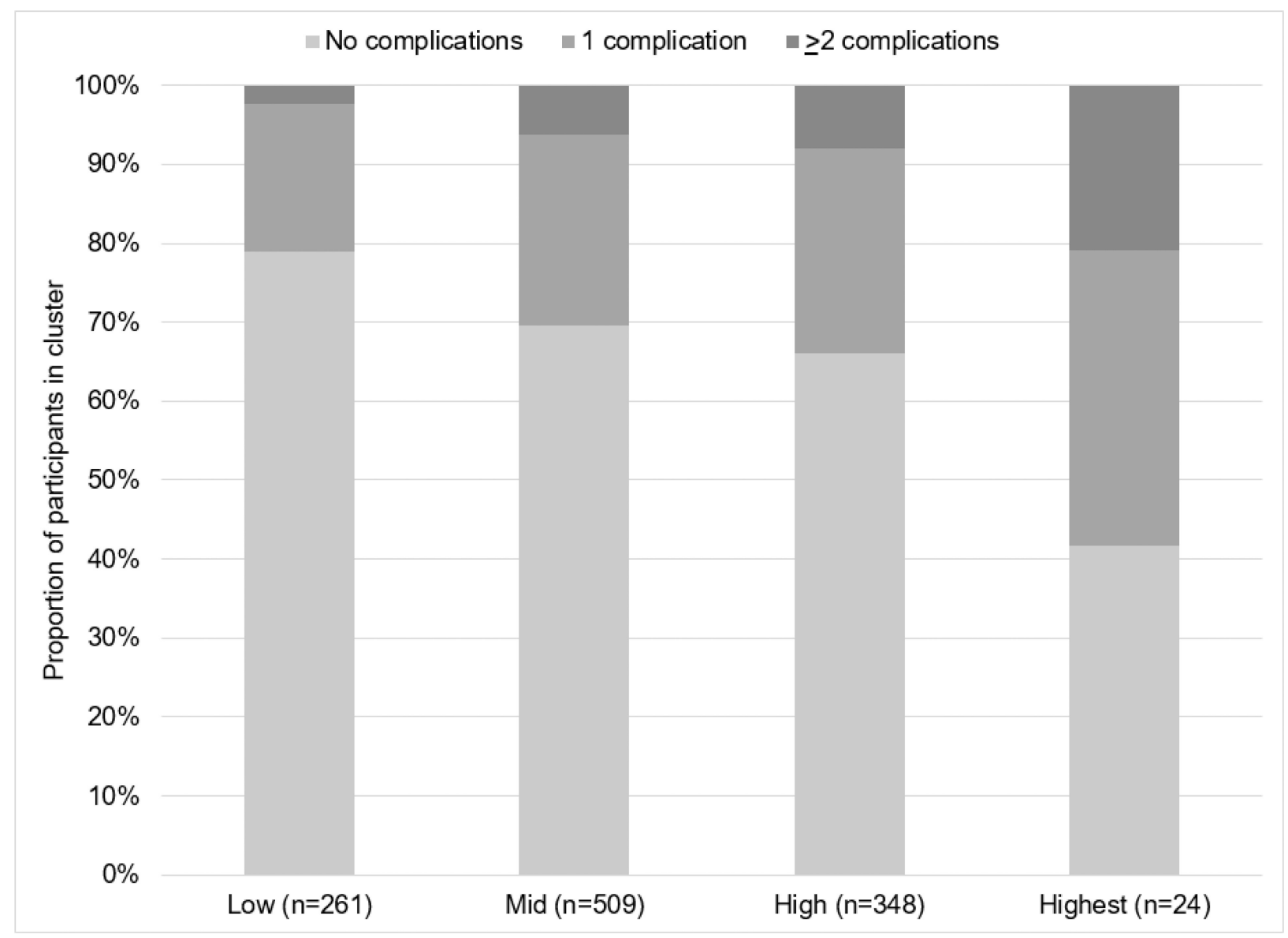

Figure 2.

Frequency of co-occurring diabetes-related complications/comorbidities among risk factor clusters of 1,142 participants with type 1 diabetes in the SEARCH for Diabetes in Youth study (2010-2015). Clusters were derived using a cluster analysis and the following variables: sex; race/ethnicity; age at the follow-up visit; baseline and follow-up duration of diabetes, hemoglobin A1c, waist to height ratio, mean arterial pressure, HDL cholesterol, and non-HDL cholesterol. Chi-Square $\mathrm{p}<0.001$ for difference in frequency of co-occurring complications between clusters. 
Table 1.

Characteristics of 1,327 participants in the SEARCH for Diabetes in Youth Study at their baseline (20012009) and follow-up (2010-2015) visits

\begin{tabular}{|c|c|c|c|c|}
\hline & & Baseline visit & & Follow up visit \\
\hline & $\mathbf{n}$ & Mean \pm SD or count $(\%)$ & $\mathbf{n}$ & Mean \pm SD or count $(\%)$ \\
\hline Female (n) & 1327 & $599(45 \cdot 1)$ & & --- \\
\hline Non-Hispanic white (n) & 1327 & $1017(76 \cdot 6)$ & & --- \\
\hline Age at diagnosis (years) & 1327 & $10 \cdot 1(3 \cdot 9)$ & & --- \\
\hline Age at visit (years) & 1327 & $10 \cdot 9(3 \cdot 9)$ & 1327 & $18 \cdot 0(4 \cdot 1)$ \\
\hline Diabetes duration (years) & 1327 & $0 \cdot 8(0 \cdot 5)$ & 1327 & $7 \cdot 8(1.9)$ \\
\hline Parental education (n) & 1311 & & 1309 & \\
\hline$<$ High school graduate & & $54(4 \cdot 1)$ & & $56(4 \cdot 3)$ \\
\hline High school graduate or higher & & $1257(95 \cdot 9)$ & & $1253(95 \cdot 7)$ \\
\hline Annual household income (n) & 1309 & & 1323 & \\
\hline$<\$ 25,000$ & & $163(12 \cdot 5)$ & & $195(14 \cdot 7)$ \\
\hline$\$ 25,000-\$ 49,999$ & & $264(20 \cdot 2)$ & & $216(16 \cdot 3)$ \\
\hline$\$ 50,000$ - $\$ 74,999$ & & $247(18.9)$ & & $206(15 \cdot 6)$ \\
\hline$>\$ 75,000$ & & $537(41 \cdot 0)$ & & $494(37 \cdot 3)$ \\
\hline Do not know/refused & & $98(7.5)$ & & $212(16 \cdot 0)$ \\
\hline Health insurance (n) & 1314 & & 1322 & \\
\hline Private & & $1055(80 \cdot 3)$ & & $961(72 \cdot 3)$ \\
\hline Medicare/Medicaid & & $206(15 \cdot 7)$ & & $250(18 \cdot 9)$ \\
\hline Other & & $33(2 \cdot 5)$ & & $65(4 \cdot 9)$ \\
\hline None & & $20(1.5)$ & & $46(3 \cdot 5)$ \\
\hline Hemoglobin A1c (\%) & 1318 & $7 \cdot 6(1.5)$ & 1326 & $9 \cdot 2(1 \cdot 8)$ \\
\hline $\mathrm{mmol} / \mathrm{mol}$ & & $60 \cdot 0(16 \cdot 1)$ & & $76 \cdot 8(20 \cdot 1)$ \\
\hline BMI z-score & 1310 & $0.5(1 \cdot 0)$ & 1327 & $0 \cdot 6(1 \cdot 0)$ \\
\hline Waist circumference $(\mathrm{cm})$ & 1296 & $65 \cdot 9(11 \cdot 4)$ & 1327 & $77.9(11 \cdot 7)$ \\
\hline Waist to height ratio & 1219 & $0.45(0.05)$ & 1327 & $0.46(0.06)$ \\
\hline Systolic blood pressure (mmHg) & 1292 & $99 \cdot 8(11 \cdot 5)$ & 1327 & $106 \cdot 7(11 \cdot 0)$ \\
\hline Diastolic blood pressure $(\mathrm{mmHg})$ & 1291 & $62 \cdot 8(10 \cdot 1)$ & 1327 & $69 \cdot 0(8 \cdot 7)$ \\
\hline Mean arterial pressure $(\mathrm{mmHg})$ & 1291 & $75 \cdot 1(9 \cdot 6)$ & 1327 & $81 \cdot 6(8 \cdot 6)$ \\
\hline Total cholesterol (mg/dl) & 1275 & $159 \cdot 8(26 \cdot 9)$ & 1325 & $170 \cdot 0(35 \cdot 6)$ \\
\hline LDL cholesterol (mg/dl) & 1275 & $91.4(22 \cdot 6)$ & 1324 & $96 \cdot 5(28 \cdot 7)$ \\
\hline HDL cholesterol (mg/dl) & 1275 & $55 \cdot 6(12 \cdot 7)$ & 1324 & $54.9(13 \cdot 4)$ \\
\hline Triglycerides (mg/dl) [median (Q1, Q3] & 1275 & $55(43,73)$ & 1325 & $74(55,105)$ \\
\hline Non-HDL cholesterol (mg/dl) & 1275 & $104 \cdot 1(25 \cdot 0)$ & 1324 & $115 \cdot 0(35 \cdot 5)$ \\
\hline
\end{tabular}

Data are mean \pm standard deviation, median (Q1, Q3) [continuous], or n (\%) [categorical]. 
Table 2.

Frequency of diabetes-related complications and comorbidities among 1,327 participants in the SEARCH for Diabetes in Youth study at an average of 7.8 years after type 1 diabetes diagnosis (2010-2015)

\begin{tabular}{|c|c|c|c|c|}
\hline & \multicolumn{2}{|c|}{ Observed } & \multirow{2}{*}{$\begin{array}{c}\text { Expected } \\
\%\end{array}$} & \multirow[b]{2}{*}{ p-value ${ }^{*}$} \\
\hline & $\mathbf{n}$ & $\%$ & & \\
\hline Number of diabetes-related complications 0 & 941 & $70 \cdot 9$ & 68.4 & 0.053 \\
\hline 1 & 308 & $23 \cdot 2$ & 27.2 & $0 \cdot 0011$ \\
\hline 2 & 62 & $4 \cdot 7$ & 4.1 & $0 \cdot 34$ \\
\hline 3 & 14 & $1 \cdot 1$ & 0.3 & $0 \cdot 0001$ \\
\hline 4 & 2 & $0 \cdot 2$ & 0.0 & $<0.0001$ \\
\hline 5 & 0 & $0 \cdot 0$ & 0.0 & --- \\
\hline Any 2 or more & 78 & $5 \cdot 9$ & 4.4 & $0 \cdot 015$ \\
\hline \multicolumn{5}{|l|}{ Individually occurring complications ${ }^{\dagger}$} \\
\hline Retinopathy & 24 & $1 \cdot 8$ & 2.9 & 0.016 \\
\hline Diabetic kidney disease & 51 & $3 \cdot 8$ & 4.2 & 0.62 \\
\hline Peripheral neuropathy & 50 & $3 \cdot 8$ & 4.2 & $0 \cdot 47$ \\
\hline Arterial stiffness & 69 & $5 \cdot 2$ & 6.3 & $0 \cdot 10$ \\
\hline Cardiovascular autonomic neuropathy & 114 & $8 \cdot 6$ & 9.6 & $0 \cdot 24$ \\
\hline \multicolumn{5}{|l|}{ Co-occurring complications ${ }^{\dagger}$} \\
\hline Retinopathy + diabetic kidney disease & 11 & $0 \cdot 8$ & 0.2 & $0 \cdot 0007$ \\
\hline Retinopathy + peripheral neuropathy & 7 & $0 \cdot 5$ & 0.2 & 0.087 \\
\hline Retinopathy + arterial stiffness & 13 & $1 \cdot 0$ & 0.3 & $0 \cdot 0016$ \\
\hline Retinopathy + cardiovascular autonomic neuropathy & 11 & $0 \cdot 8$ & 0.5 & $0 \cdot 15$ \\
\hline Diabetic kidney disease + peripheral neuropathy & 5 & $0 \cdot 4$ & 0.3 & $0 \cdot 89$ \\
\hline Diabetic kidney disease + arterial stiffness & 12 & $0 \cdot 9$ & 0.5 & 0.059 \\
\hline Diabetic kidney disease + cardiovascular autonomic neuropathy & 10 & $0 \cdot 8$ & 0.7 & $0 \cdot 90$ \\
\hline Peripheral neuropathy + arterial stiffness & 8 & $0 \cdot 6$ & 0.5 & $0 \cdot 65$ \\
\hline Peripheral neuropathy + cardiovascular autonomic neuropathy & 15 & $1 \cdot 1$ & 0.7 & $0 \cdot 11$ \\
\hline Arterial stiffness + cardiovascular autonomic neuropathy & 24 & $1 \cdot 8$ & 1.0 & 0.015 \\
\hline
\end{tabular}


Table 3.

Characteristics of risk factor clusters among 1,142 participants with type 1 diabetes in the SEARCH for Diabetes in Youth study

\begin{tabular}{|c|c|c|c|c|c|}
\hline & Low & Mid & High & Highest & p-value* \\
\hline Size of cluster (n) ${ }^{\dagger}$ & $261(22 \cdot 9)$ & $509(44 \cdot 6)$ & $348(30 \cdot 5)$ & $24(2 \cdot 1)$ & \\
\hline Female (n) & $100(38 \cdot 3)$ & $218(42 \cdot 8)$ & $175(50 \cdot 3)$ & $10(41 \cdot 7)$ & 0.0064 \\
\hline Non-Hispanic white (n) & $217(83 \cdot 1)$ & $395(77 \cdot 6)$ & $257(73 \cdot 9)$ & $15(62 \cdot 5)$ & 0.0017 \\
\hline Age at diagnosis (years) & $10 \cdot 5(3 \cdot 7)$ & $10 \cdot 5(3 \cdot 6)$ & $10 \cdot 9(3 \cdot 6)$ & $9 \cdot 7(2 \cdot 6)$ & $0 \cdot 30^{f}$ \\
\hline \multicolumn{6}{|l|}{ Baseline visit (2001-2009) } \\
\hline Age (years) & $11 \cdot 3(3 \cdot 7)$ & $11 \cdot 4(3 \cdot 5)$ & $11 \cdot 7(3 \cdot 6)$ & $10 \cdot 9(2 \cdot 7)$ & $0 \cdot 47^{*}$ \\
\hline Diabetes duration (years) & $0 \cdot 7(0 \cdot 5)$ & $0 \cdot 8(0 \cdot 6)$ & $0 \cdot 8(0 \cdot 5)$ & $1 \cdot 1(0 \cdot 9)$ & $0 \cdot 018$ \\
\hline Hemoglobin A1c (\%) & $7 \cdot 4(1 \cdot 3)$ & $7 \cdot 7(1 \cdot 5)$ & $7 \cdot 7(1.7)$ & $8 \cdot 1(1 \cdot 8)$ & $0 \cdot 088$ \\
\hline $\mathrm{mmol} / \mathrm{mol}$ & $57 \cdot 6(13 \cdot 7)$ & $60 \cdot 3(16 \cdot 0)$ & $60 \cdot 8(18 \cdot 4)$ & $64 \cdot 8(20 \cdot 0)$ & $0 \cdot 088$ \\
\hline Waist to height ratio & $0.44(0 \cdot 04)$ & $0 \cdot 45(0 \cdot 05)$ & $0.47(0.06)$ & $0 \cdot 47(0 \cdot 05)$ & $<0.0001$ \\
\hline Mean arterial pressure $(\mathrm{mmHg})$ & $74 \cdot 8(9 \cdot 2)$ & $76 \cdot 0(9 \cdot 6)$ & $76 \cdot 5(9 \cdot 1)$ & $70 \cdot 3(11 \cdot 2)$ & 0.0051 \\
\hline HDL cholesterol (mg/dl) & $58 \cdot 6(13 \cdot 2)$ & $56 \cdot 5(12 \cdot 4)$ & $52 \cdot 2(12 \cdot 4)$ & $58.4(14.9)$ & $<0.0001$ \\
\hline Non-HDL cholesterol (mg/dl) & $78 \cdot 4(12 \cdot 6)$ & $104 \cdot 5(16 \cdot 9)$ & $120 \cdot 6(26 \cdot 1)$ & $125 \cdot 6(23 \cdot 9)$ & $<0.0001$ \\
\hline \multicolumn{6}{|l|}{ Follow-up visit (2010-2015) } \\
\hline Age (years) & $18 \cdot 0(4 \cdot 2)$ & $18 \cdot 4(3 \cdot 9)$ & $18 \cdot 9(3 \cdot 8)$ & $17 \cdot 8(2 \cdot 9)$ & $0 \cdot 021$ \\
\hline Diabetes duration (years) & $7 \cdot 4(1.9)$ & $7 \cdot 8(1.9)$ & $8.0(1.9)$ & $8 \cdot 0(2 \cdot 0)$ & $0 \cdot 0028$ \\
\hline Hemoglobin A1c (\%) & $8.5(1.5)$ & $9 \cdot 0(1 \cdot 7)$ & $9 \cdot 7(1.9)$ & $11 \cdot 8(2 \cdot 0)$ & $<0.0001$ \\
\hline $\mathrm{mmol} / \mathrm{mol}$ & $69 \cdot 8(16 \cdot 7)$ & $74 \cdot 6(18 \cdot 5)$ & $82 \cdot 8(21 \cdot 2)$ & $105 \cdot 2(21 \cdot 7)$ & $<0.0001$ \\
\hline Waist to height ratio & $0.44(0.05)$ & $0.46(0 \cdot 06)$ & $0.49(0.07)$ & $0.50(0 \cdot 05)$ & $<0.0001$ \\
\hline Mean arterial pressure $(\mathrm{mmHg})$ & $80 \cdot 0(8 \cdot 0)$ & $82 \cdot 6(8 \cdot 6)$ & $82.4(8.4)$ & $84 \cdot 4(10 \cdot 1)$ & 0.0003 \\
\hline HDL cholesterol (mg/dl) & $58 \cdot 7(12 \cdot 8)$ & $54 \cdot 4(13 \cdot 1)$ & $51 \cdot 4(12 \cdot 3)$ & $50 \cdot 4(12 \cdot 4)$ & $<0.0001$ \\
\hline Non-HDL cholesterol (mg/dl) & $79 \cdot 0(12 \cdot 9)$ & $105 \cdot 7(12 \cdot 2)$ & $147 \cdot 9(21 \cdot 5)$ & $245 \cdot 5(33 \cdot 5)$ & $<0.0001$ \\
\hline Health insurance (n) & & & & & $<0.0001^{\frac{t}{t}}$ \\
\hline Private & $211(80 \cdot 8)$ & $386(76 \cdot 1)$ & $222(64 \cdot 2)$ & $9(37.5)$ & \\
\hline Medicare/Medicaid & $34(13 \cdot 0)$ & $87(17 \cdot 2)$ & $83(24 \cdot 0)$ & $11(45 \cdot 8)$ & \\
\hline Other & $10(3 \cdot 8)$ & $23(4 \cdot 5)$ & $19(5 \cdot 5)$ & $1(4 \cdot 2)$ & \\
\hline None & $6(2 \cdot 3)$ & $11(2 \cdot 2)$ & $22(6 \cdot 4)$ & $3(12 \cdot 5)$ & \\
\hline Parental education (n) & & & & & $0.057 \%$ \\
\hline$<$ High school graduate & $6(2 \cdot 3)$ & $24(4 \cdot 8)$ & $20(5 \cdot 8)$ & $1(5 \cdot 0)$ & \\
\hline High school graduate or higher & $253(97 \cdot 7)$ & $476(95 \cdot 2)$ & $326(94 \cdot 2)$ & $19(95 \cdot 0)$ & \\
\hline Annual household income (n) & & & & & $0.0020^{* t}$ \\
\hline$<\$ 25,000$ & $26(10 \cdot 0)$ & $73(14 \cdot 4)$ & $69(19.9)$ & $6(25 \cdot 0)$ & \\
\hline$\$ 25,000-\$ 49,999$ & $41(15 \cdot 8)$ & $72(14 \cdot 2)$ & $66(19 \cdot 0)$ & $5(20 \cdot 8)$ & \\
\hline$\$ 50,000$ - $\$ 74,999$ & $36(13 \cdot 8)$ & $85(16 \cdot 7)$ & $44(12 \cdot 7)$ & $2(8 \cdot 3)$ & \\
\hline$>\$ 75,000$ & $125(48 \cdot 1)$ & $185(36 \cdot 4)$ & $111(32 \cdot 0)$ & $5(20 \cdot 8)$ & \\
\hline Do not know/refused & $32(12 \cdot 3)$ & $93(18 \cdot 3)$ & $57(16 \cdot 4)$ & $6(25 \cdot 0)$ & \\
\hline
\end{tabular}


Data are $\mathrm{n}(\%)$ or mean (SD). Clusters were derived using a cluster analysis and the following variables: sex; race/ethnicity; age at the follow-up visit; baseline and follow-up duration of diabetes, hemoglobin A1c, waist to height ratio, mean arterial pressure, HDL cholesterol, and non-HDL cholesterol

P-values reflect Chi-Square tests (categorical) or Kruskal-Wallis tests (continuous).

${ }^{\dagger}$ Percentages reflect the proportion of the full sample $(\mathrm{n}=1,142)$; all other percentages reflect the proportion of the cluster.

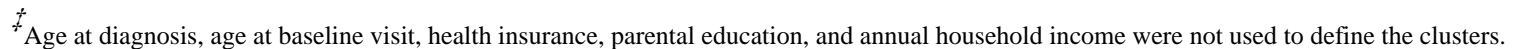
Sample sizes for health insurance, parental education, and annual household income varied (1138, 1125, and 1139, respectively). 